ARTICLE

\title{
High-performance n-type black phosphorus transistors with type control via thickness and contact-metal engineering
}

\author{
David J. Perello ${ }^{1}$, Sang Hoon Chae ${ }^{1}$, Seunghyun Song ${ }^{1} \&$ Young Hee Lee $e^{1,2,3}$
}

Recent work has demonstrated excellent p-type field-effect switching in exfoliated black phosphorus, but type control has remained elusive. Here, we report unipolar n-type black phosphorus transistors with switching polarity control via contact-metal engineering and flake thickness, combined with oxygen and moisture-free fabrication. With aluminium contacts to black phosphorus, a unipolar to ambipolar transition occurs as flake thickness increases from 3 to $13 \mathrm{~nm}$. The $13-\mathrm{nm}$ aluminium-contacted flake displays graphene-like symmetric hole and electron mobilities up to $950 \mathrm{~cm}^{2} \mathrm{~V}^{-1} \mathrm{~s}^{-1}$ at $300 \mathrm{~K}$, while a $3 \mathrm{~nm}$ flake displays unipolar n-type switching with on/off ratios greater than $10^{5}\left(10^{7}\right)$ and electron mobility of $275(630) \mathrm{cm}^{2} \mathrm{~V}^{-1} \mathrm{~s}^{-1}$ at $300 \mathrm{~K}(80 \mathrm{~K})$. For palladium contacts, $\mathrm{p}$-type behaviour dominates in thick flakes, while $2.5-7 \mathrm{~nm}$ flakes have symmetric ambipolar transport. These results demonstrate a leap in n-type performance and exemplify the logical switching capabilities of black phosphorus.

\footnotetext{
${ }^{1}$ IBS Center for Integrated Nanostructure Physics, Institute for Basic Science, Sungkyunkwan University, Suwon 440-746, Korea. ${ }^{2}$ Department of Energy Science, Sungkyunkwan University, Suwon 440-746, Korea. ${ }^{3}$ Department of Physics, Sungkyunkwan University, Suwon 440-746, Korea. Correspondence and requests for materials should be addressed to D.J.P. (email: dperello16@gmail.com) or to Y.H.L. (email: leeyoung@skku.edu).
} 
U ltrathin black phosphorus (BP), colloquially referred to as phosphorene, is a layered allotrope of phosphorus with a highly anisotropic and thickness-dependent band structure $^{1-5}$. In contrast to semiconducting transition metal dichalcogenides with typically large bandgap and small mobility, or zero gap and high-mobility monolayer graphene, BP has both high mobility ${ }^{1}$ (in bulk) and a bandgap ranging from 0.3 to $0.39 \mathrm{eV}$ in bulk to $\sim 1.5-2 \mathrm{eV}$ for monolayer ${ }^{3-7}$. Currently, thin layers can be accessed only from exfoliation of bulk crystalline BP samples, as no chemical vapor deposition (CVD) or epitaxial synthesis method exists. Despite this limitation, initial reports for exfoliated flakes between 2 and $20 \mathrm{~nm}$ thick have demonstrated that the material can be used as a high-performance p-type conducting channel with room temperature hole mobilities $\left(\mu_{\mathrm{FE}}\right)$ of $10^{2}-10^{3} \mathrm{~cm}^{2} \mathrm{~V}^{-1} \mathrm{~s}^{-1}$ (refs 8-14). Intrinsically however, theory predicts that pristine BP displays no dominant preference to carrier type. Thus far, only p-type unipolar and p-type dominant ambipolar transport has been observed due to suppressed electron transport, resulting from a combination of oxygen and moisture exposure ${ }^{15-21}$. To date, no n-type transistor operation or clear mechanism of type control has been reported ${ }^{8}$.

In the following, we demonstrate that $\mathrm{BP}$ can serve as a high-performance n-type, ambipolar, or p-type transistor channel dependent on both flake thickness and contact metal. Theoretical layer dependence of the valence and conduction band edges ${ }^{3}$ ( $E_{\mathrm{C}}$ and $E_{\mathrm{V}}$, respectively) is exploited to fabricate high-performance n-type FETs with electron mobilities of $10^{2}-10^{3} \mathrm{~cm}^{2} \mathrm{~V}^{-1} \mathrm{~s}^{-1}$ at $300 \mathrm{~K}$ using $\mathrm{Al}$ as a Schottky-type contact. With $\mathrm{Al}$ contacts, ultrathin 3-nm flakes show the most promise for use in logic circuits, with a combined $I_{\text {on }} / I_{\text {off }}>10^{5}$ $\left(10^{7}\right)$ and electron mobility of $275(630) \mathrm{cm}^{2} \mathrm{~V}^{-1} \mathrm{~s}^{-1}$ at $300 \mathrm{~K}$ (80 K). Al-BP Schottky-field-effect transistors (Schottky FETs) are contrasted with $\mathrm{Pd}-\mathrm{BP}$ samples with lower contact resistance. With Pd contacts, FETs are channel resistance-dominant devices and more intrinsic properties of the flakes can be extracted. These devices display a p-type to symmetric ambipolar transition when the thickness of the BP is reduced. The influence of dielectric coating, orientation and dual gating on carrier type and device performance is further investigated.

\section{Results}

Al-contacted n-type FETs. Transport measurements of BP-FETs are performed in both 4-terminal and 2-terminal configurations to extract contact and channel properties of the BP-FETs. Figure 1a shows a 6-terminal hall bar fabricated on a 3-nm thick flake with $\mathrm{Al}(10 \mathrm{~nm}) / \mathrm{Cr}(3 \mathrm{~nm}) / \mathrm{Au}(20 \mathrm{~nm})$ contact-metal stack. Al was chosen as the contact material due to its low work function $\left(\Phi_{\mathrm{M}} \sim 4.0-4.3 \mathrm{eV}\right)$ and lengthy history as a lowresistance $n$-type contact to semiconductors like silicon ${ }^{22}$. In contrast to heavily doped silicon, thin intrinsic BP is highly conductive without a fixed majority carrier. Therefore, with Al contacts, $I_{\mathrm{ds}}-V_{\mathrm{bg}}$ (d,s,bg subscripts denote the drain, source, and back gate, respectfully) measurements display clear Schottky-FET behaviour. This is shown at $300 \mathrm{~K}$ in Fig. 1b, where unipolar behaviour and an extremely large but unsaturated (4-terminal) electron carrier mobility $\mu_{\mathrm{FE}}=275 \mathrm{~cm}^{2} \mathrm{~V}^{-1} \mathrm{~s}-1$ at $V_{\mathrm{bg}}=70 \mathrm{~V}$ is observed. Hole transport is suppressed such that $I_{\mathrm{on}} / I_{\mathrm{off}} \sim 10^{5}$ at $300 \mathrm{~K}$, which is further enhanced to $10^{7}$ at $80 \mathrm{~K}$, as shown in Fig. 1c. Notably, peak electron mobility (4-terminal) is enhanced to $630 \mathrm{~cm}^{2} \mathrm{~V}^{-1} \mathrm{~s}^{-1}$ at $80 \mathrm{~K}\left(V_{\mathrm{bg}}=70 \mathrm{~V}\right)$, while band-edge mobility at $V_{\mathrm{bg}}=9 \mathrm{~V}$ increases from 1 to $290 \mathrm{~cm}^{2} \mathrm{~V}^{-1} \mathrm{~s}^{-1}$. Hole mobilities show no improvement with decreased temperature. The subthreshold swing (SS), which is described by $\mathrm{SS}=\ln (10) k T\left(1+C_{\mathrm{BP}} / C_{\mathrm{SiO}_{2}}\right)$, shows a temperature-dependent reduction from $9 \mathrm{~V}$ per decade at $300 \mathrm{~K}$ to $2.5 \mathrm{~V}$ per decade at $80 \mathrm{~K}$. Substituting into the above, the depletion capacitance of the $3.5 \mathrm{~nm}$ thick $\mathrm{BP}$ is $C_{\mathrm{BP}}=1.62 \mu \mathrm{F} \mathrm{cm}^{-2}$.

To understand the influence of the contact resistance, we compare 4-terminal measurements with 2-terminal results on the same BP sample. At $50 \mathrm{~V}$, two terminal peak mobility of $94 \mathrm{~cm}^{2} \mathrm{~V}^{-1} \mathrm{~s}^{-1}$ compares with the 4-terminal $150 \mathrm{~cm}^{2} \mathrm{~V}^{-1} \mathrm{~s}^{-1}$ electron mobility at $300 \mathrm{~K}$. The $50 \%$ increase in the mobility results from the large contact resistance with $\mathrm{Al}$. Improvements to a

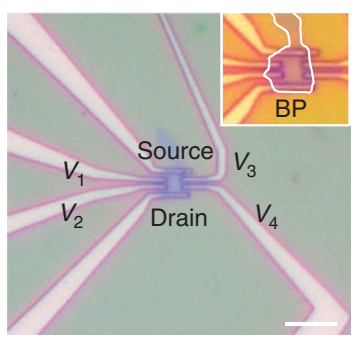

C

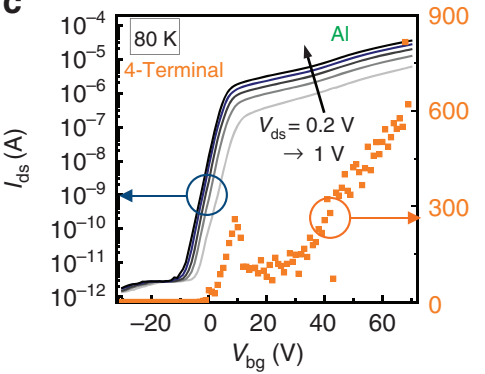

b

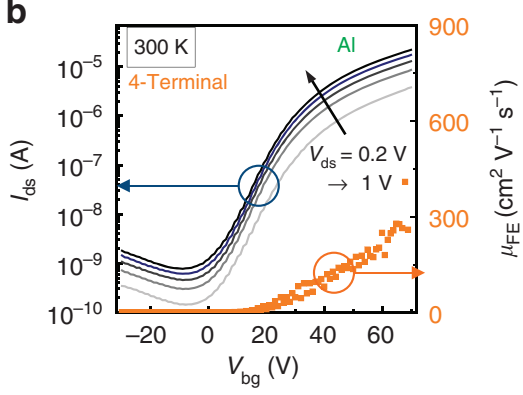

d

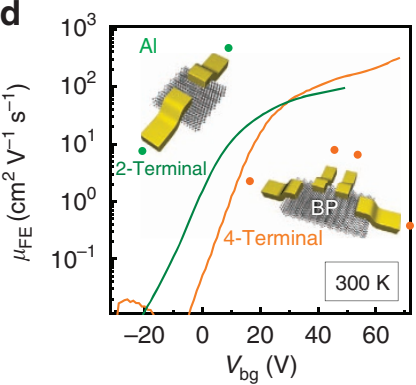

Figure 1 | High-performance $\mathbf{n}$-type transistors with Al contacts. (a) Optical image of the device for four-probe measurements. Scale bar is $6 \mu \mathrm{m}$. (b) $I_{\mathrm{ds}}$ showing $\mathrm{n}$-type operation with $I_{\mathrm{on}} / I_{\mathrm{off}} \sim 10^{5}$ at $300 \mathrm{~K}$. The $\mathrm{n}$-type 4-terminal-calculated $\mu_{\mathrm{FE}}$ at $300 \mathrm{~K}$ is $275 \mathrm{~cm}^{2} \mathrm{~V}^{-1} \mathrm{~s}^{-1}$. (c) $I_{\mathrm{ds}}$ showing n-type operation with $I_{\mathrm{on}} / I_{\mathrm{off}}>10^{7}$ at $80 \mathrm{~K}$. The $\mathrm{n}$-type 4-terminal mobility is $\mu_{\mathrm{FE}} \sim 630 \mathrm{~cm}^{2} \mathrm{~V}^{-1} \mathrm{~s}^{-1}$ at $80 \mathrm{~K}$ with no sign of saturation. (d) Comparison of 2-terminal and 4-terminal field-effect mobilities. Contact resistance is the source of this difference. 
n-type contact resistance while retaining suppressed hole transport will need to be addressed in further studies. All samples with $\mathrm{Al}$ contacts displayed n-type behaviour (other examples can be found in Supplementary Fig. 1).

Confirmation of Schottky barrier origins of the n-type behaviour was then established by performing activation energy measurements (Supplementary Fig. 2) to extract equilibrium barrier heights $\left(\Phi_{\mathrm{B}}\right)$ at $V_{\mathrm{ds}}=0 \mathrm{~V}$ for different gate potentials. Results for a 2-terminal n-type Al-contacted 6-nm thick BP flake are detailed in Fig. 2. For $V_{\mathrm{bg}} \geq-10 \mathrm{~V}$, excellent fit with pure thermionic emission is achieved. Additionally, by linearly fitting the dependence of $\Phi_{\mathrm{B}}$ on $V_{\mathrm{bg}}$, we find that $\partial \Phi_{\mathrm{B}} / \partial V_{\mathrm{bg}} \approx 6 \mathrm{mV}\left(\Phi_{\mathrm{B}}\right) / V_{\mathrm{bg}}$. This equates to $S S=10 \mathrm{~V}$ per decade at $300 \mathrm{~K}$, which is in agreement with the $I_{\mathrm{ds}}-V_{\mathrm{bg}}$ raw data in Fig. 2 and further demonstrates that the switching is contact dominant.

With $V_{\mathrm{bg}}<-10 \mathrm{~V}, \Phi_{\mathrm{B}}$ displays weak or no $V_{\mathrm{ds}}$ dependence which results from an increased off-state channel resistance or a subsequent increase in field emission current as $V_{\text {bg }}$ pushes the Fermi level into the valence band. Tunnelling is more likely in this case, since the flake is 6-nm thick and the $I_{\mathrm{ds}}-V_{\mathrm{bg}}$ sweep in Fig. 2 shows an exponential increase in current with $V_{\mathrm{ds}}$ at $V_{\mathrm{bg}}<-30 \mathrm{~V}$. For thinner devices, as in Fig. 1, tunnelling is prevented by a larger bandgap and thicker depletion region. The $V_{\text {bg }}$ dependence found here mirrors the Schottky barrier analysis of p-type devices in two recent reports ${ }^{23,24}$, although barrier heights here are at equilibrium which is in contrast to the Arrhenius analysis ${ }^{23}$.

We next investigated the flake thickness dependence of transport with $\mathrm{Al}$ contacts on a separate elongated Hall bar geometry fabricated on the thickness-tapered edge of a larger flake (Fig. 3a). Optical image of the tapered flake with decorated electrode locations is shown in Fig. 3a.

BP thickness varies from $3.5 \mathrm{~nm}$ between probes $V_{34}\left(V_{67}\right)$, to $8 \mathrm{~nm}$ between probes $V_{23}\left(V_{56}\right)$, and up to $13 \mathrm{~nm}$ between $V_{12}$. The thickness is not constant across the surface and thus an average estimate was used. Atomic force microscopy (AFM) is not ideal for determining the precise number of BP layers, but the exact height is not critical in obtaining general trends of transport, which can be determined by the relative differences in thickness on a single flake. 4-Terminal transport properties were measured at $300 \mathrm{~K}$ (Fig. 3b) and $80 \mathrm{~K}$ (Fig. 3d) to contrast sheet resistance $\left(R_{\mathrm{s}}\right)$ and carrier mobilities for different thicknesses. These measurements show that $3.5-\mathrm{nm}$ flakes retain the unipolar n-type transport similar to the device with a $3-\mathrm{nm}$

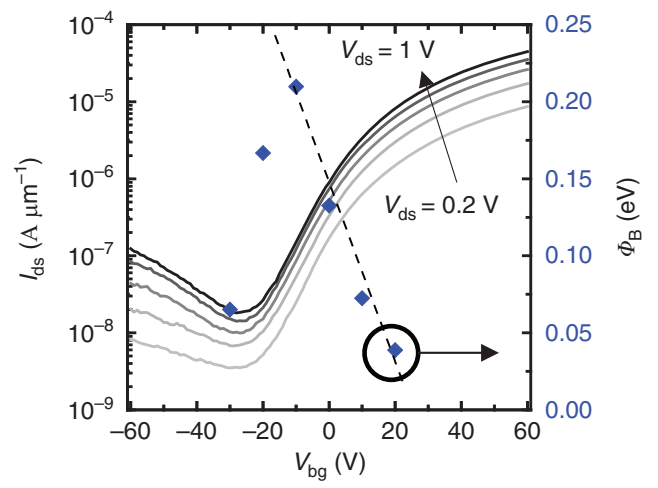

Figure 2 | Al-BP Schottky barrier measurement. $l_{\mathrm{ds}}-V_{\mathrm{bg}}$ at $300 \mathrm{~K}$ for a 6-nm thick flake overlaid with the Schottky barrier height $\Phi_{\mathrm{B}}$, which was extracted via activation energy measurements. The barrier at $V_{\mathrm{bg}}=-30 \mathrm{~V}$ represents a tunneling barrier while $V_{\mathrm{bg}} \geq-10 \mathrm{~V}$ displays excellent fit with pure thermionic emission. flake in Fig. 1. As the flake thickness increases, electron mobility improves while a more noticeable increase in hole mobility occurs. For a 13-nm flake, transport is nearly ambipolar with an unsaturated and nearly symmetric electron and hole mobility of $\sim 950 \mathrm{~cm}^{2} \mathrm{~V}^{-1} \mathrm{~s}^{-1}$.

Due to the extreme instability of the BP, defining a precise mesa via an additional lithography and etching step was not performed. Time-to-measurement, air exposure and increased edge diffusion of oxygen and water molecules were deemed more problematic to the thickness-dependent relationship than mesa patterning ${ }^{16,18}$. Mobilities for the non-etched sample (Fig. 3a) were calculated based on an ideal assumption of no current spread across the flake due to the presence of Schottky contacts at all the voltage probe arms $\left(V_{1}-V_{7}\right)$. This assumption can lead to overestimation of mobilities if the contact barriers are not large. In comparison, for samples with a defined mesa, a large positive threshold voltage $\left(V_{\mathrm{T}}\right)$ shift typically occurs, preventing mobility

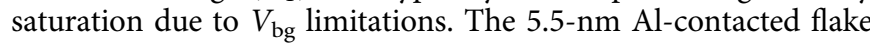
in Fig. 3b (gray) is one example and is shifted by $-35 \mathrm{~V}$ to coincide with the non-etched flake. In comparing etched with non-etched samples, even without saturation, the peak mobility $\left(250 \mathrm{~cm}^{2} \mathrm{~V}^{-1} \mathrm{~s}^{-1}\right)$ of the etched sample fits within the thickness trend established for the non-etched flake in Fig. 3a. Therefore, the mobilities of the non-etched flake are not overtly overestimated. Mobility magnitude is also consistent with Fig. 1, which does not have such an unorthodox shape. Further discussion of mobility calculations can be found in Supplementary Fig. 3.

The thickness dependence of electron mobility is modelled via Matthiessen's rule in Fig. $3 \mathrm{c}: \mu_{\mathrm{FE}}^{-1}=\mu_{\mathrm{Bulk}}^{-1}+\mu_{\text {Surface }}^{-1}(t)$, where $t$ is the thickness of the flake measured from AFM measurement. We extract $\mu_{\mathrm{Bulk}}=2.6 \times 10^{3} \mathrm{~cm}^{2} \mathrm{~V}^{-1} \mathrm{~s}^{-1}$ as $t$ goes to infinity and $\mu_{\mathrm{Mono}}=94 \mathrm{~cm}^{2} \mathrm{~V}^{-1} \mathrm{~s}^{-1}$ at the monolayer limit. The monolayer estimate is extracted by assuming an AFM-measured thickness of $t_{\text {Mono }}=1.5 \mathrm{~nm}$. A unit of $1.5 \mathrm{~nm}$ is chosen as an upper bound of monolayer thickness since the $\mathrm{BP} / \mathrm{SiO}_{2}$ has $\sim 1 \mathrm{~nm}$ roughness in AFM scans, and the BP bulk layer spacing is $0.55 \mathrm{~nm}$. The roughness and sample-tip interaction difference between $\mathrm{SiO}_{2}$ and BP can lead to a significant overestimate of thickness. The observed mobility reduction and significant surface scattering in ultrathin layers are a direct consequence of stronger interlayer interaction. Carriers in BP are highly delocalized perpendicular to the plane direction, which contrasts graphene and many transition metal dichalcogenides where layer interaction is governed strictly by weak van der Waal's interaction ${ }^{25-27}$. The axis perpendicular to the basal plane in BP has a well-defined band structure and experimentally verified carrier mobility that is larger than the in-plane puckered direction ${ }^{1}$. Thus, surface imperfections are a more significant contribution to carrier scattering than in other multilayered van der Waal's materials. The extrapolated bulk value here is also larger than experimental estimates from previous experiments in thicker films ${ }^{10}$, but is similar to recent theoretical estimates ${ }^{28}$, cryogenic measurements on hBN encapsulated samples ${ }^{12,29}$ and bulk measurements ${ }^{1}$.

The tapered flake was also measured at $80 \mathrm{~K}$ for temperaturerelated mobility changes. At $80 \mathrm{~K}$, due to the reduced distribution of thermal energy for carriers, $I_{\text {on }} / I_{\text {off }}$ increases for all thicknesses and off-state conductivity is suppressed. Only the thickest $13 \mathrm{~nm}$ region shows a small increase in band-edge mobility (marked by filled square in Fig. 3d). The temperature-independent n-type mobility of the thinner areas is consistent with increased surface scattering due to non-uniform flake thickness. This is in contrast with Fig. 1, where the device was fabricated on a flake with uniform thickness and therefore has a noticeable mobility increase from 300 to $80 \mathrm{~K}$. Crystalline bulk BP and other thin p-type devices ${ }^{12,29}$ characterized in the literature have also shown 

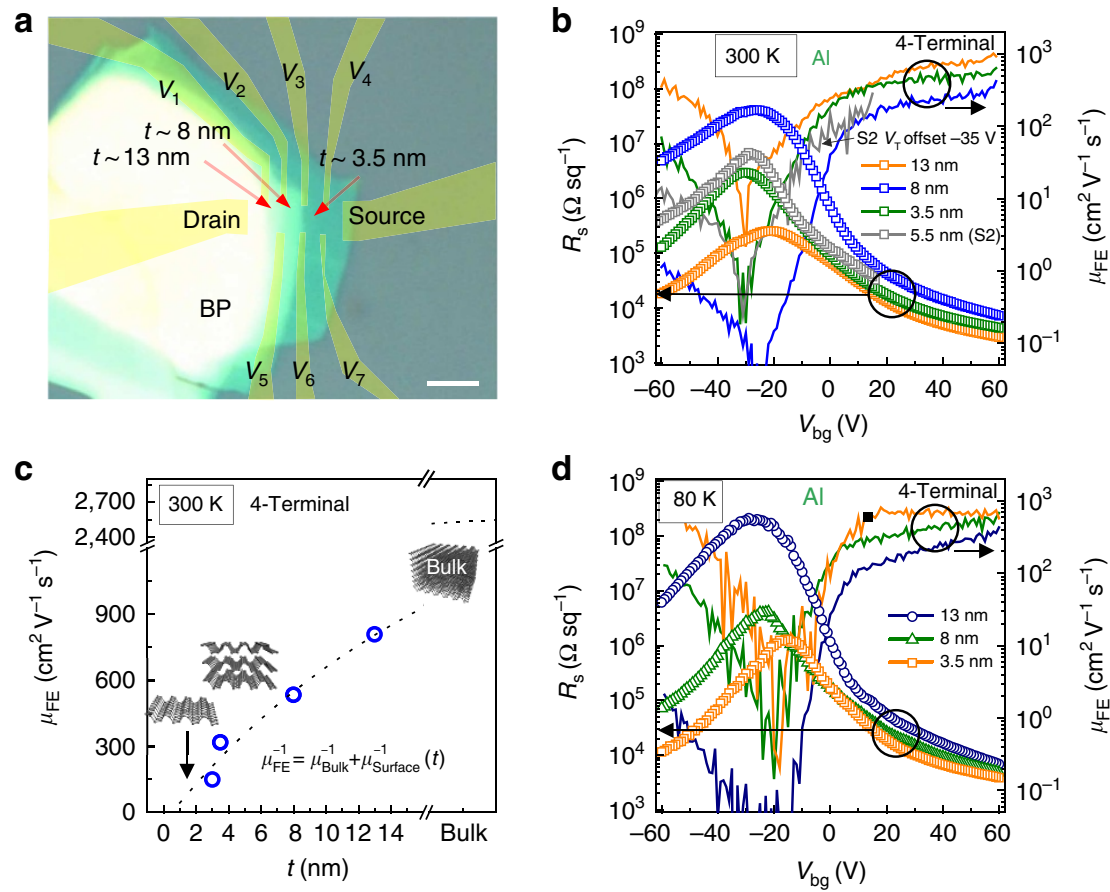

Figure 3 | BP thickness dependences of Al-contacted devices. (a) Optical image of the BP flake (before processing) used for testing layer dependences of transport properties with Al contacts. Yellow shaded region shows locations of electrodes (Supplementary Fig. 3). Scale bar, $3 \mu \mathrm{m}$. (b) $R_{\mathrm{s}}$ (open points) and $\mu_{\mathrm{FE}}$ (solid lines) from 4-terminal measurements at $300 \mathrm{~K}$. (c) $\mu_{\mathrm{FE}}$ at $300 \mathrm{~K}$ and $V_{\mathrm{bg}}=50 \mathrm{~V}$ fit to Matthiessen's rule. From fitting, $\mu_{\mathrm{Bulk}}=2.6 \times 10^{3} \mathrm{~cm}^{2} \mathrm{~V}-1 \mathrm{~s}^{-1}$ and estimated monolayer surface scattering is limited to $94 \mathrm{~cm}^{2} \mathrm{~V}^{-1} \mathrm{~s}^{-1}$. (d) $R_{\mathrm{s}}$ (open points) and $\mu_{\mathrm{FE}}$ (solid lines) from 4-terminal measurements at $80 \mathrm{~K}$. The $n$-type to ambipolar conversion is observed as the flake thickness increases. Mobility increases with increasing thickness, with $n$ - and $p$-type $\mu_{\mathrm{FE}} \sim 9.5 \times 10^{2} \mathrm{~cm}^{2} \mathrm{~V}^{-1} \mathrm{~s}^{-1}$ at $80 \mathrm{~K}$ for $13 \mathrm{~nm}$ thick region $\left(V_{1,2}\right.$ in $\left.\mathbf{a}\right)$.

a large increase in carrier mobility at low temperature, although the data shown in Fig. 1 is the first evidence of localized bandedge mobility increase.

Pd-contacted ambipolar FETs. Before summarizing the $\mathrm{Al}$ Schottky-FET transport, we consider Pd, a high-work-function metal $\left(\Phi_{\mathrm{m}}=5.0+\mathrm{eV}\right)$ as a contact to BP. With $\mathrm{Pd}(10 \mathrm{~nm}) /$ $\mathrm{Cr}(3 \mathrm{~nm}) / \mathrm{Au}(20 \mathrm{~nm})$ contacts, ambipolar and hole-dominant operation is consistently observed for all samples in our experiments and an example is shown in Fig. 4a (see more in Supplementary Figs 4-6). Reducing temperature to $80 \mathrm{~K}$ improved 2-terminal electron mobility in Pd-contacted samples by $50 \mathrm{~cm}^{2} \mathrm{~V}^{-1} \mathrm{~s}^{-1}$, while 2 -terminal hole mobility increases by only $8 \mathrm{~cm}^{2} \mathrm{~V}^{-1} \mathrm{~s}^{-1}$ in the measured 7 -nm thick flake.

The dependence of transport on flake thickness is explored in Fig. 4b. By increasing BP flake thickness stepwise from 2.5 to $14.5 \mathrm{~nm}$, the $I_{\mathrm{ds}}-V_{\mathrm{bg}}$ transitions from nearly symmetric ambipolar to p-type dominant, as shown in Fig. 4b,c. This coincides with a sharp positive (right) shift of the $V_{\mathrm{T}}$, and a rapid decrease of $I_{\text {on }} / I_{\text {off }}$ with increased thickness. Mobility also increases with thickness, reaching a saturated (2-terminal) $140 \mathrm{~cm}^{2} \mathrm{~V}^{-1} \mathrm{~s}^{-1}$ for thickness $t=14.5 \mathrm{~nm}$ at $300 \mathrm{~K}$. Furthermore, total suppression of electron transport is not observed for Pd-contacted FETs of any thickness, but more consistent hole-dominant transport occurs in thicker flakes.

Summarizing thickness and contact-metal dependences. The thickness and metal dependences of BP-FETs are summarized in Table 1. Control of the dominant carrier type is a function of the Schottky barrier at the contact, akin to carbon nanotubes ${ }^{30,31}$. Aluminium works as a good n-type contact for all BP thicknesses because the conduction band minimum only weakly depends on flake thickness. Using ref. 5, 3-5 layer BP has $E_{\mathrm{C}} \sim 4.1 \mathrm{eV}$ and the bulk can be extrapolated to $\sim 4.2 \mathrm{eV}$. $E_{\mathrm{C}}$ matches well to the $\mathrm{Al}$ work function of $4.0-4.3 \mathrm{eV}$. For $V_{\mathrm{bg}}>>V_{\mathrm{T}}$ (electron-type transport), increased mobility, increased $I_{\mathrm{ds}}$ and reduced $R_{\mathrm{s}}$ with decreased temperature are evidence of a reduced Schottky barrier. Conversely, for $V_{\mathrm{bg}}<<V_{\mathrm{T}}$ (hole-type transport), a large Schottky barrier exists between $\mathrm{Al}$ and ultrathin flakes $(3-3.5 \mathrm{~nm})$. Since the 4-terminal measurements show this hole carrier suppression in each of 3-8 $\mathrm{nm}$ devices, we suggest that the $\mathrm{Al}$ contact depletion region extends across the extent of the 1.5-3 $\mu \mathrm{m}$ channels, holding the BP Fermi level to the conduction band minimum throughout the channel. This is further evidenced by the indiscernible n-type threshold voltage change for all thicknesses in Fig. 3d. Transport becomes more ambipolar in thicker flakes, indicating the hole-type Schottky barrier is reduced due to reduction of the bandgap. This is schematically shown in the thickness-dependent band diagrams for Al-BP in Fig. 5. Note that 2-terminal devices with $\mathrm{Al}$ contacts to thicker BP also have ambipolar-type transport and reduced $I_{\text {on }} / I_{\text {off }}$ (Supplementary Fig. 7). Therefore, control of the hole-type Schottky barrier is the critical factor in transport, and the unipolar n-type to ambipolar transition results from a reduction of the barrier height as flake thickness is increased.

Meanwhile, Pd contacts have previously been shown to reduce hole-type contact resistance compared with other metals ${ }^{32}$. Our results indicate that contact resistance of $\mathrm{Pd}$ to ultrathin $\mathrm{BP}$ $(2.5 \mathrm{~nm})$ is an order of magnitude smaller than that of the channel resistance (Supplementary Fig. 4). We hypothesize that the low contact resistance is related to $\operatorname{Pd}_{x} \mathrm{P}_{y}$ alloy formation at the contact ${ }^{33}$, but more rigorous study is required. This channel resistance naturally decreases as the $\mathrm{BP}$ thickness increases. Therefore, as BP thickness increases, the Pd-BP contact resistance will become more significant relative to the channel 

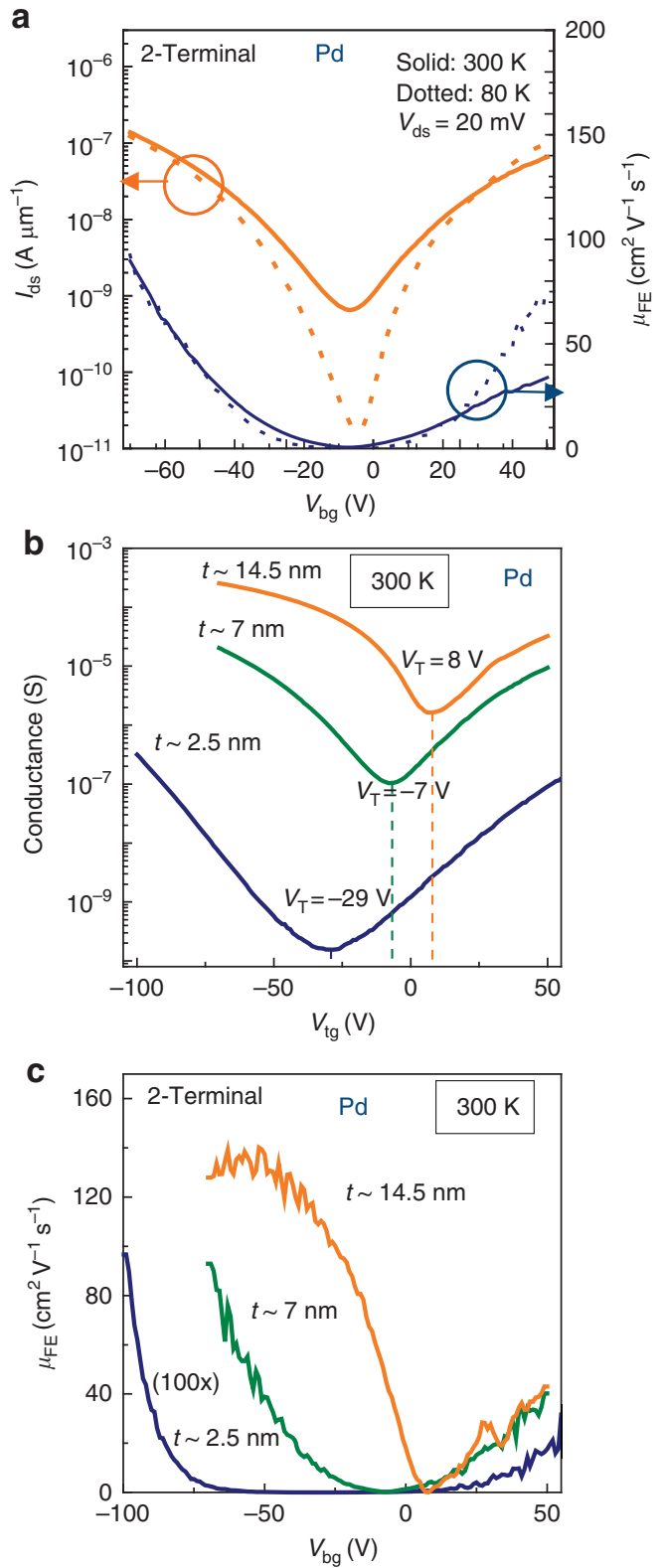

Figure 4 | Thickness dependences of Pd-contacted BP. (a) Ambipolar BP-FET ( $7 \mathrm{~nm}$ thickness) measured at 300 and $80 \mathrm{~K}$ and the corresponding mobilities. (b) Thickness ( $t$ )-dependent $V_{T}$ shift and ambipolar to unipolar conversion, consistent with decreased BP work function with increasing thickness. (c) Large p-type enhancement of field-effect mobility in thick flakes, while thin flakes display reduced and type-symmetric mobilities.

resistance. This is reflected experimentally in Fig. 4, where $I_{\mathrm{ds}}$ increases, and a transition from the intrinsic ambipolar to contact-influenced p-type is observed. This factor also likely contributes to the temperature independence of mobility for the ambipolar 2-terminal measurements in Fig. 4a, contrasting 4 -terminal results on previous p-type samples ${ }^{12,29}$. Although previous work also demonstrated a mobility increase with 2-terminal measurements and $\mathrm{Pd}$ as a contact, the results are slightly different. One obvious difference is that our sample fabrication was done in a glove box, whereas in the previous approach exfoliation was done in air and shows more dominant p-type characteristics. The resulting ambipolarity of our Pd-contacted samples results in almost equal resistance for both electrons and holes, which also contrasts the p-type dominant
Table 1 | Type control summary by thickness and contact metal.

\begin{tabular}{|c|c|c|c|}
\hline & \multicolumn{3}{|c|}{ BP thickness } \\
\hline & $2.5-5.5 \mathrm{~nm}$ & $7-8 \mathrm{~nm}$ & $13-14.5 \mathrm{~nm}$ \\
\hline Al contacts & Unipolar n-type & Unipolar n-type & Ambipolar \\
\hline Pd contacts & Ambipolar & $\begin{array}{l}\text { Ambipolar p-type } \\
\text { dominant }\end{array}$ & Unipolar n-type \\
\hline
\end{tabular}

a

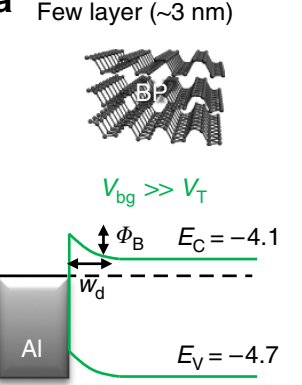

b Thick (13-15 $\mathrm{nm})$

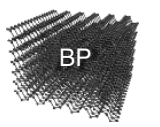

$V_{\mathrm{bg}} \gg V_{\mathrm{T}}$
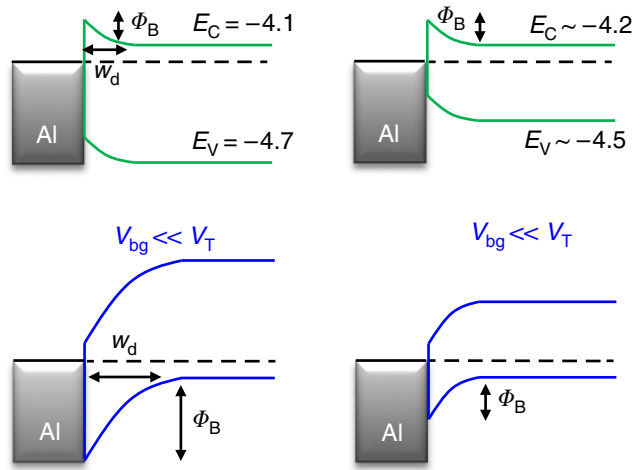

Figure 5 | Band alignment at aluminium-BP interface. Schematic band diagrams based on previously calculated $E_{V}$ and $E_{C}$ (ref. 3) for (a) few layer ( $3 \mathrm{~nm})$ and (b) thick BP flakes with Al contacts (13-15 nm). The insensitivity of $E_{C}$ to thickness change and makes $\mathrm{Al}$ a great n-type contact but very high-resistance $p$-type contact with a large depletion width $\left(w_{d}\right)$ for thin BP. Thicker samples have small Schottky barriers for both $\mathrm{n}$ and p-transport, permitting ambipolar operation.

phenomena in previous work ${ }^{32}$. While trivial at $300 \mathrm{~K}$, at $80 \mathrm{~K}$ a parasitic contact resistance can mask the expected increase in BP carrier mobility. The presence of symmetric ambipolar operation in different devices is a clear sign of the quality of the samples. The p-type dominance results from the built-in potential at the contacts proportional to $\Phi_{\mathrm{M}}-E_{\mathrm{V}}$. This induces a large band bending, whereby excess hole carriers are donated from the Pd to the BP channel. This hole doping is also found in some ultrathin films (Supplementary Fig. 6), but is mostly dominant in thicker flakes. As the thickness of BP increases, a threshold shift is observed in Fig. $4 \mathrm{~b}$, which is attributed to the change of $E_{\mathrm{V}}$ from $-4.85 \mathrm{eV}$ in trilayer to an estimated -4.5 in bulk ${ }^{3,5}$.

Dual gating and directional transport. To compare with the above contact metal-based control of the device polarity, we investigate the effects of dual gating and deposition of $\mathrm{Al}_{2} \mathrm{O}_{3}$ dielectric on channel-dominant Pd-contacted samples. In particular, we examine the influence of a vertical displacement field $D=\left(C_{\mathrm{t}} V_{\mathrm{tg}}-C_{\mathrm{b}} V_{\mathrm{bg}}\right) / 2$ and surface passivation on majority carrier type and mobilities. An optical image of the sample investigated is shown in Fig. 6a. Using the top gate potential to vary the Fermi level, it can be seen that the presence of $\mathrm{Al}_{2} \mathrm{O}_{3}$ and induced displacement field yields no type conversion or polarity changes. Instead, symmetric hole and electron ambipolarity is retained in Fig. 6 b, as in the $7 \mathrm{~nm}$ back-gated results in Fig. 4 , which is the same 
a
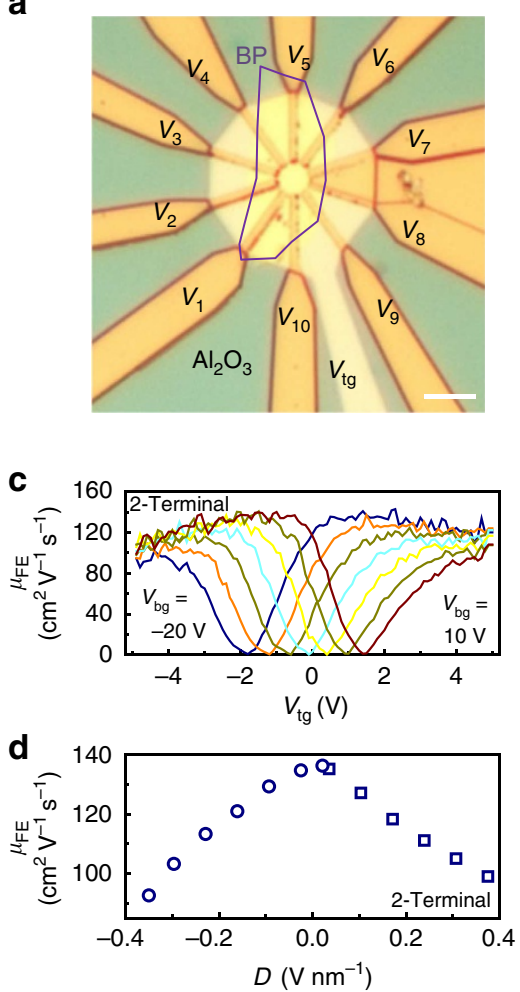
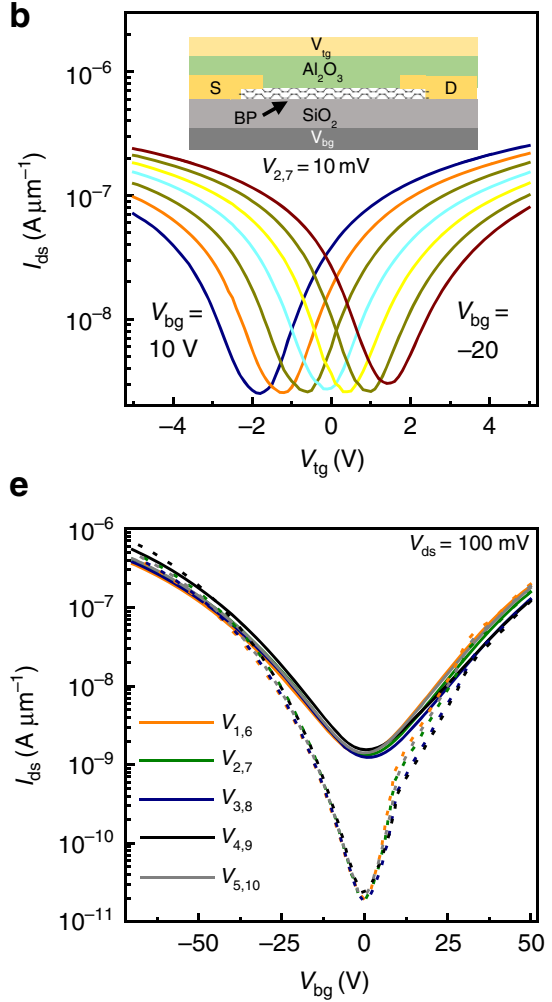

Figure 6 | Ambipolar dual-gated BP-FETs. (a) Optical image of 7-nm thick dual-gated (top and bottom) device. (b) Fully symmetric dual-gated FET operation. The applied displacement field has no influence on device polarity. Scale bar, $5 \mu \mathrm{m}$. (c) 2 -Terminal mobilities measured via the $V_{\mathrm{tg}}$ sweep. Mobility saturation is observed and electron-hole transport symmetry is retained, although peak values increased by $45 \mathrm{~cm}^{2} V^{-1} \mathrm{~s}^{-1} \mathrm{compared}$ with pre- $\mathrm{Al}_{2} \mathrm{O}_{3}$ measurement in Fig. 4c. Inset shows the device schematic from a. $\mathrm{Al}_{2} \mathrm{O}_{3}$ dielectric thickness is $50 \mathrm{~nm}$ and $\mathrm{SiO}_{2}$ thickness is $300 \mathrm{~nm}$. (d) Peak mobility for electrons (squares) and hole (circles) branches of the curves in c, plotted as a function of $D$, normalized to a thickness of $7 \mathrm{~nm}$. (e) No clear directional dependences are observed for $I_{\mathrm{ds}}$ at both $300 \mathrm{~K}$ (solid) and $80 \mathrm{~K}$ (dotted).

device before $\mathrm{Al}_{2} \mathrm{O}_{3}$ deposition. However, due to all-around gate coupling and presence of a high- $\kappa$ dielectric, a $45 \mathrm{~cm}^{2} \mathrm{~V}^{-1} \mathrm{~s}^{-1}$ increase in peak hole mobility from reduced surface scattering ${ }^{34}$, and mobility saturation are observed in Fig. 6c. Peak electron and hole carrier mobility symmetry $\left(140 \mathrm{~cm}^{2} \mathrm{~V}^{-1} \mathrm{~s}^{-1}\right)$ is also retained for all applied displacement fields, as shown in Fig. 6d. A displacement field induces a carrier concentration gradient across the $\mathrm{BP}$, which is effectively a change in the channel thickness contributing to the conduction, similar to the width modulation of the strongly inverted region of a traditional buried metal-oxidesemiconductor FET. With all-around gating of BP and $D=0$, peak field-effect mobility is maximized, as shown in Fig. $6 \mathrm{~d}$. This bodes well for future devices; because back-gated Al-contacted BP results can be further improved. In particular, thicker flakes will benefit most since all-around gating is more effective in inverting the carrier type for more layers in the channel. By stepping $V_{\mathrm{bg}}$, the vertical displacement field across the $\mathrm{BP}$ varies, producing a nearly linear shift of $V_{\mathrm{T}}$ without a dominant polarity conversion or change in off-state current. This result indicates that an electric field generated either via a top gate, surface dielectric or surface functionalization (doping) is not a promising way to control a carrier type in flakes as thin as $7 \mathrm{~nm}$. The influence of surface functionalization, dielectric growth or a displacement field on thinner BP flakes remains an open question.

Lastly, to ensure that the comparisons between flakes and metal contact types are not an artifact of device flake alignment, we examine directional dependences of carrier transport in $\mathrm{Pd}$-contacted devices. This angle dependence is examined before and after $\mathrm{Al}_{2} \mathrm{O}_{3}$ gate dielectric growth. As shown in Fig. 6e, no angle dependence on carrier type is observed at 300 and $80 \mathrm{~K}$ in the $I_{\mathrm{ds}}-V_{\mathrm{bg}}$ sweeps. The angle dependence as a function of the top gate is found in Supplementary Fig. 5. This hints to anisotropic transport, but we are unable to quantify whether the small differences are related to angle. Absence of a clear directional influence is in good contrast with theoretical prediction or previous experimental report of anisotropic transport ${ }^{1,11,28}$. We presume that transport in the current samples is therefore dominated by extrinsic factors, not the intrinsic effective mass anisotropy present in BP. Similarly, the thickness dependences of mobility in any of the results of this communication are also not inadvertently directionally related. The quality of the samples fabricated here is evidenced by reduced hysteresis (Supplementary Fig. 6) when compared with the early reports in the literature. Improved sample quality is due to the moisturefree glove box fabrication and/or coverage by a top gate dielectric $^{18,20,35}$. This procedure induces less residual coulomb scattering centres and trap sites in the surrounding dielectrics.

\section{Discussion}

We have demonstrated BP transistors with unipolar n-type, ambipolar and unipolar p-type characteristics by varying contactmetal and flake thickness dependences. With Al contacts, we demonstrated the first high-performance unipolar n-type $\mathrm{BP}$ transistors with electron mobilities ranging from $10^{2}$ to $10^{3} \mathrm{~cm}^{2} \mathrm{~V}^{-1} \mathrm{~s}^{-1}$ and $I_{\mathrm{on}} / I_{\text {off }}>10^{5}$ at room temperature. A flake thickness dependence of dominant carrier type was also observed, which is due to the existence of a Schottky barrier at the Al-BP contact. 
The excellent switching characteristics and possibility of symmetric electron and hole mobilities is promising for complementary metal-oxide-semiconductor (CMOS) integration, wherein mobility is often a limiting factor in switching speed. While the current samples are still limited by extrinsic factors such as surface roughness and contact resistance, we have demonstrated that dual gating can improve performance. The current weakness of BP is the environmental instability and lack of a scalable CVD or epitaxial growth method. If progress on these issues is successful, the ability to vary transistor operation from n-type, ambipolar or p-type via modulation of the Fermi level (electrostatic gating), Schottky barrier (contact metal, channel length and electrostatic gating) and bandgap (flake thickness) makes BP a very dynamic and useful material for logic circuits.

\section{Methods}

Sample preparation. BP flakes (99.998\%) were purchased from smart-elements.com and stored in an inert atmosphere. Exfoliation and subsequent fabrication procedures where $\mathrm{BP}$ was unprotected (for example, uncovered with PMMA or $\mathrm{Al}_{2} \mathrm{O}_{3}$ ) were performed in a glove box with $\mathrm{H}_{2} \mathrm{O}$ and $\mathrm{O}_{2}$ concentration < 1 p.p.m. After exfoliating flakes onto an $\mathrm{Ar}$-annealed $\mathrm{Si} / \mathrm{SiO}_{2}(300 \mathrm{~nm})$ wafer and finding suitable clean locations with an optical microscope, electrodes were patterned by electron beam lithography and either $\mathrm{Al} / \mathrm{Cr} / \mathrm{Au}$ or $\mathrm{Pd} / \mathrm{Cr} / \mathrm{Au}$ was evaporated for contact electrodes. Total fabrication was completed in $<24 \mathrm{~h}$ in all cases. Until high vacuum condition was achieved, all procedures were performed with limited or no light exposure, including storage of the flakes. $\mathrm{Al}_{2} \mathrm{O}_{3}$ was grown using $\mathrm{Al}_{2} \mathrm{O}_{3}$ dielectric with chamber temperature of $120^{\circ} \mathrm{C}$. The chamber was thoroughly cooled before growth to prevent any initial oxidation of the BP during transfer from inert environment. The oxide thickness of $50 \mathrm{~nm}$ corresponds to 500 cycles with trimethyl aluminium precursor.

Field-effect mobilities. Measurements were performed in a vacuum probe station in low $10^{-6}$ torr vacuum with a $\mathrm{LN}_{2}$ feed through for $80 \mathrm{~K}$ measurements. The typical planar capacitance model was utilized, $\mu_{\mathrm{FE}}=W g_{\mathrm{m}} / L V_{\mathrm{ds}} C_{\mathrm{ox}}$, where $V_{\mathrm{ds}}$ is the applied voltage for 2-terminal and the measured probe voltages for the 4-terminal case. The $C_{\mathrm{b}}=11.6 \mathrm{nF} \mathrm{cm}^{-2}$ for the $300-\mathrm{nm}$ thick $\mathrm{SiO}_{2}$ back gate oxide and $C_{\mathrm{t}}=100 \mathrm{nF} \mathrm{cm}^{-2}$ for the $50-\mathrm{nm}$ thick $\mathrm{Al}_{2} \mathrm{O}_{3}$ top gate dielectric. Supplementary Figure $5 \mathrm{~d}$ contains further details regarding $C_{\mathrm{b}}$ and $C_{\mathrm{t}}$. Geometry for the 4-terminal mobility calculations was defined by the central $\mathrm{BP}$ region between the electrodes. Details, example region and possible estimation errors are outlined in Supplementary Fig. 3.

Schottky barrier measurement. Temperature-dependent $I_{\mathrm{ds}}-V_{\mathrm{ds}}$ measurements were performed while also stepping the back-gate potential $V_{\mathrm{bg}} . V_{\mathrm{ds}}$-dependent $\Phi_{\mathrm{B}}$ was calculated from fitting the slope of the Richardson plot $\ln \left(I_{\mathrm{ds}} / T^{2}\right)$ versus $1 / k T$. Equilibrium Schottky barrier height, $\Phi_{\mathrm{B}}\left(V_{\mathrm{ds}}\right)=0$, was then extracted by plotting $\Phi_{\mathrm{B}}\left(V_{\mathrm{ds}}\right)$ versus $V_{\mathrm{ds}}^{1 / 2}$ and extrapolating the $V_{\mathrm{ds}}=0$ intercept.

\section{References}

1. Narita, S. et al. Far-infrared cyclotron resonance absorptions in black phosphorus single crystals. J. Phys. Soc. Jpn. 52, 3544-3553 (1983).

2. Rudenko, A. N. \& Katsnel'son, M. I. Quasiparticle band structure and tightbinding model for single- and bilayer black phosphorus. Phys. Rev. B 89, 201408 (2014)

3. Cai, Y., Zhang, G. \& Zhang, Y.-W. Layer-dependent band alignment and work function of few-layer phosphorene. Sci. Rep. 4, 6677 (2014).

4. Guan, J., Zhu, Z. \& Tománek, D. Phase coexistence and metal-insulator transition in few-layer phosphorene: a computational study. Phys. Rev. Lett. 113, 046804 (2014)

5. Tran, V., Soklaski, R., Liang, Y. \& Yang, L. Layer-controlled band gap and anisotropic excitons in few-layer black phosphorus. Phys. Rev. B 89, 235319 (2014).

6. Liang, L. et al. Electronic bandgap and edge reconstruction in phosphorene materials. Nano Lett. 14, 6400-6406 (2014).

7. Asahina, H., Shindo, K. \& Morita, A. Electronic structure of black phosphorus in self-consistent pseudopotential approach. J. Phys. Soc. Jpn. 51, 1193-1199 (1982).

8. Du, Y., Liu, H., Deng, Y. \& Ye, P. D. Device perspective for black phosphorus field-effect transistors: contact resistance, ambipolar behavior, and scaling. ACS Nano 8, 10035-10042 (2014).

9. Liu, H. et al. Phosphorene: an unexplored 2D semiconductor with a high hole mobility. ACS Nano 8, 4033-4041 (2014).
10. Li, L. et al. Black phosphorus field-effect transistors. Nat. Nano 9, 372-377 (2014).

11. Xia, F., Wang, H. \& Jia, Y. Rediscovering black phosphorus as an anisotropic layered material for optoelectronics and electronics. Nat. Commun. 5, 4458 (2014).

12. Nathaniel, G. et al. Gate tunable quantum oscillations in air-stable and high mobility few-layer phosphorene heterostructures. 2D Mater. 2, 011001 (2015).

13. Cao, Y. et al. Quality heterostructures from two dimensional crystals unstable in air by their assembly in inert atmosphere. Preprint at http://arxiv.org/abs/ 1502.03755 (2015).

14. Chen, X. et al. High quality sandwiched black phosphorus heterostructure and its quantum oscillations. Preprint at http://arxiv.org/abs/1412.1357 (2014).

15. Wang, H. et al. Black phosphorus radio-frequency transistors. Nano Lett. 14, 6424-6429 (2014).

16. Island, J. O. et al. Environmental instability of few-layer black phosphorus. $2 D$ Mater. 2, 011002 (2015)

17. Doganov, R. et al. Accessing the transport properties of pristine few-layer black phosphorus by van der Waals passivation in inert atmosphere. Preprint at http://arxiv.org/abs/1412.1274 (2014).

18. Wood, J. D. et al. Effective passivation of exfoliated black phosphorus transistors against ambient degradation. Nano Lett. 14, 6964-6970 (2014).

19. Andres, C.-G. et al. Isolation and characterization of few-layer black phosphorus. 2D Mater. 1, 025001 (2014).

20. Koenig, S. P., Doganov, R. A., Schmidt, H., Castro Neto, A. H. \& Özyilmaz, B. Electric field effect in ultrathin black phosphorus. Appl. Phys. Lett. 104, 103106 (2014).

21. Favron, A. et al. Exfoliating pristine black phosphorus down to the monolayer: photo-oxidation and electronic confinement effects. Preprint at http://arxiv.org/ abs/1408.0345 (2014)

22. Matlow, S. L. \& Ralph, E. L. Ohmic aluminum-n-type silicon contact. J. Appl. Phys. 30, 541-543 (1959).

23. Das, S. et al. Tunable transport gap in phosphorene. Nano Lett. 14, 5733-5739 (2014).

24. Kamalakar, M. V. et al. Low Schottky barrier black phosphorus field-effect devices with ferromagnetic tunnel contacts. Small 11, 2209-2216 (2015).

25. Das, S. \& Appenzeller, J. Screening and interlayer coupling in multilayer $\mathrm{MoS}_{2}$. Phys. Status Solidi RRL 7, 268-273 (2013).

26. Rydberg, H. et al. Van der Waals density functional for layered structures. Phys. Rev. Lett. 91, 126402 (2003).

27. McKenzie, R. H. \& Moses, P. Incoherent interlayer transport and angulardependent magnetoresistance oscillations in layered metals. Phys. Rev. Lett. 81, 4492-4495 (1998).

28. Qiao, J., Kong, X., Hu, Z.-X., Yang, F. \& Ji, W. High-mobility transport anisotropy and linear dichroism in few-layer black phosphorus. Nat. Commun. 5, 4475 (2014).

29. Li, L. et al. Quantum oscillations in black phosphorus two-dimensional electron gas. Preprint at http://arxiv.org/abs/1411.6572 (2014).

30. Perello, D. J. et al. Anomalous schottky barriers and contact band-to-band tunneling in carbon nanotube transistors. ACS Nano 4, 3103-3108 (2010).

31. Zhang, Z. et al. Doping-free fabrication of carbon nanotube based ballistic CMOS devices and circuits. Nano Lett. 7, 3603-3607 (2007).

32. Das, S., Demarteau, M. \& Roelofs, A. Ambipolar phosphorene field effect transistor. ACS Nano 8, 11730-11738 (2014).

33. Okamoto, H. The P-Pd (phosphorus-palladium) system. J. Phase Equilib. 15, 58-61 (1994).

34. Ong, Z.-Y. \& Fischetti, M. V. Mobility enhancement and temperature dependence in top-gated single-layer $\mathrm{MoS}_{2}$. Phys. Rev. B 88, 165316 (2013).

35. Han, L. et al. The effect of dielectric capping on few-layer phosphorene transistors: tuning the Schottky barrier heights. IEEE Electr. Device Lett. 35, 795-797 (2014).

\section{Acknowledgements}

This work was supported by IBS-R011-D1.

\section{Author contributions}

D.J.P. performed experimental design and planning, measurements, data analysis, and manuscript preparation. S.S performed AFM analysis. S.H.C. organized data and helped write the paper. Y.H.L. designed the problem, performed data analysis and helped with manuscript preparation. All authors discussed the results and commented on the manuscript.

\section{Additional information}

Supplementary Information accompanies this paper at http://www.nature.com/ naturecommunications 
Competing financial interests: The authors declare no competing financial interests.

Reprints and permission information is available online at http://npg.nature.com/ reprintsandpermissions/

How to cite this article: Perello, D. J. et al. High-performance n-type black phosphorus transistors with type control via thickness and contact-metal engineering. Nat. Commun. 6:7809 doi: 10.1038/ncomms8809 (2015). (c) (i) This work is licensed under a Creative Commons Attribution 4.0 International License. The images or other third party material in this article are included in the article's Creative Commons license, unless indicated otherwise in the credit line; if the material is not included under the Creative Commons license, users will need to obtain permission from the license holder to reproduce the material. To view a copy of this license, visit http://creativecommons.org/ licenses/by/4.0/ 


\section{Corrigendum: High-performance n-type black phosphorus transistors with type control via thickness and contact-metal engineering}

David J. Perello, Sang Hoon Chae, Seunghyun Song \& Young Hee Lee

Nature Communications 6:7809 doi: 10.1038/ncomms8809 (2015); Published 30 Jul 2015; Updated 28 Jan 2016

In Fig. 3 of this article, there are a number of errors in the colours used for the data points and curves. In Fig. $3 b$, the blue data should be green, referring to a thickness of ' $3.5 \mathrm{~nm}$ ', and the green data should be blue, referring to a thickness of ' $8 \mathrm{~nm}$ '. In Fig. $3 \mathrm{~d}$, the blue data should be green and refer to a thickness of ' $3.5 \mathrm{~nm}$ ', the green data should be blue and refer to a thickness of ' $8 \mathrm{~nm}$ ' and the orange data should refer to a thickness of ' $13 \mathrm{~nm}$ '.

In Table 1, the Pd contacts on 13-14.5 nm of BP were 'Unipolar p-type', not 'Unipolar n-type'.

The correct version of Fig. 3 and Table 1 appear below.

a

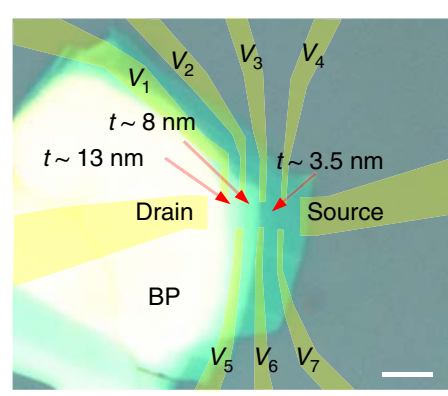

C

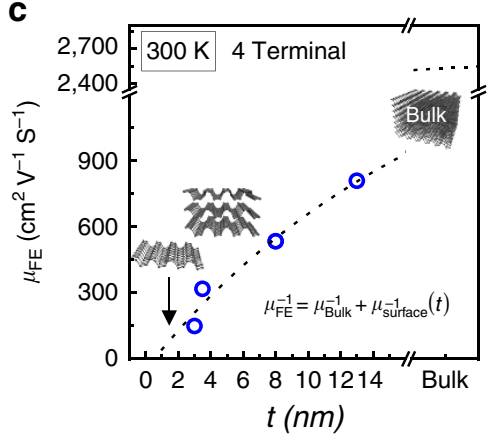

b

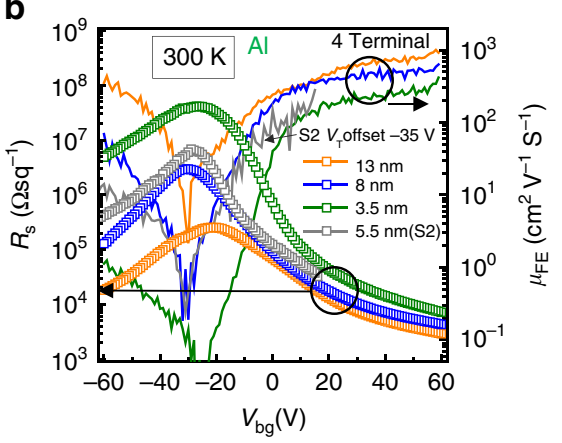

d

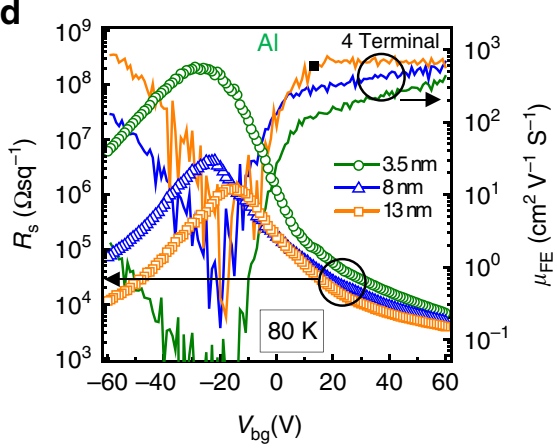

Figure 3 


\section{Table 1 | Type control summary by thickness and contact metal.}

\begin{tabular}{|cccc|} 
& \multicolumn{3}{c}{ BP thickness } \\
\cline { 2 - 4 } & $\mathbf{2 . 5 - 5 . 5} \mathbf{~ n m}$ & $\mathbf{7 - 8} \mathbf{~ n m}$ & $\mathbf{1 3 - 1 4 . 5} \mathbf{~ n m}$ \\
\hline Al contacts & $\begin{array}{c}\text { Unipolar n-type } \\
\text { Ambipolar }\end{array}$ & $\begin{array}{c}\text { Unipolar } \mathrm{n} \text {-type } \\
\text { Ambipolar } \text { p-type dominant }\end{array}$ & $\begin{array}{c}\text { Ambipolar } \\
\text { Unipolar } \text {-type }\end{array}$ \\
\hline
\end{tabular}

(c) (i) This work is licensed under a Creative Commons Attribution 4.0 International License. The images or other third party material in this article are included in the article's Creative Commons license, unless indicated otherwise in the credit line; if the material is not included under the Creative Commons license, users will need to obtain permission from the license holder to reproduce the material. To view a copy of this license, visit http://creativecommons.org/licenses/by/4.0/ 